\title{
Multiparameter Fusion Decision Routing Algorithm for Energy-Constrained Wireless Sensor Networks
}

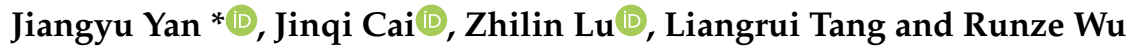 \\ State Key Laboratory of Alternate Electrical Power System with Renewable Energy Sources, \\ North China Electric Power University, Beijing 102206, China; 1172201352@ncepu.edu.cn (J.C.); \\ 1182201241@ncepu.edu.cn (Z.L.); tangliangrui@163.com (L.T.); wurz@ncepu.edu.cn (R.W.) \\ * Correspondence: yabjy@ncepu.edu.cn; Tel.: +86-010-61772520
}

Received: 6 March 2020; Accepted: 13 April 2020; Published: 16 April 2020

\begin{abstract}
For energy-limited wireless sensor networks (WSNs), we propose a multiparameter fusion decision routing (MPFDR) algorithm in this study. This algorithm gives a comprehensive account of the residual energy and forward distance, single-hop transmission ratio, cache queue, and energy equilibrium degree. It calculates the routing evaluation parameters of the forward neighbors, realizing a multidirectional reflection of the network status. Simultaneously, combined with the defined routing selection strategy based on the parameter contribution degree and fuzzy contribution degree, the fusion contribution degree of each forward neighbor is obtained. Then, the node with the most considerable fusion contribution degree is selected as the next hop. Finally, the performance of the MPFDR algorithm is simulated and compared with other algorithms. Simulation results indicate that our algorithm has good congestion control ability in energy-limited wireless sensor networks and can significantly reduce the packet loss rate and average hops.
\end{abstract}

Keywords: Wireless sensor networks; multiparameter fusion decision; routing algorithm; routing optimization parameter; fusion contribution degree

\section{Introduction}

\subsection{Background and Motivation}

Wireless sensor networks (WSNs) are considered one of the most valuable technologies in the 21st century. A wireless sensor network is constituted of a number of sensor nodes for information collection and comprehensive surveillance. Each node takes a perception, communication, and computation function. They communicate with each other in a multihop and self-organization manner through wireless radio. In the current development situation, WSNs have found wide research applications in intelligent life [1-4], industrial and agricultural production [5-8], disaster prevention and control [9-11], military applications [12], etc. In many applications, WSNs play an irreplaceable role. In particular, the advent of the Internet of Things era has accelerated the development and implementation of wireless sensor network technology and gradually led modern life to intelligence and convenience.

In the monitoring area, the sensors acquire data, transmit them to the destination node through multihop mode, and finally deliver them to the user using the external network. At the same time, with the miniaturization of sensor nodes, there are higher requirements for WSNs in terms of the capacities of data acquisition, computing, storage, and energy consumption. More importantly, in a WSN, the routing protocol plans the route of data transmission and is one of the keys to collecting information successfully. Therefore, the design of the routing protocol is a hot topic. 


\subsection{Related Work}

In WSNs, data transmission follows the preset rules and standards. A routing protocol is responsible for selecting the best path between any two nodes in WSNs. At present, researchers have thrown a diversity of routing algorithms from different points of view. A WSN node is usually low-cost and equipped low-capacity battery. It is hard to replace the battery after the node was deployed. Energy routing protocols have always held excellent research potential. There are some traditional routing algorithms, such as the Maximum Residual Energy algorithm (MRE) [13], the Minimum Path-Energy Consumption algorithm (MPEC) [14], and the Minimum Transmission Energy Consumption algorithm (MTEC) [15]. The MRE algorithm takes residual energy of each node as the routing standard and forwards the data to the node with more residual energy. The MPEC algorithm constructs the path with the minimum energy consumption from the data source to the anchor, cutting down the end-to-end transmission energy. The MTEC strives to reduce the energy of transmission and selects the node with the least single-hop energy consumption to relay data. Although the routing protocol considers the influence of the energy factor, the performance of these traditional algorithms is more prominent in terms of some aspects of network performance. However, this causes other aspects to be unacceptable because of a remarkably one-sided pursuit of energy. For example, the MRE algorithm constructs a longer routing path to bypass low-energy nodes and extend the network lifetime. Therefore, it can deteriorate the mean energy consumption and delay in wireless sensor networks.

Therefore, different from the above traditional energy routings with a single consideration, some improved algorithms are proposed in [16-28]. Among them, the authors of [16-19] propose a routing selection strategy combining the residual energy and nodes distance. In [16], for the limited energy problem and as the nodes around the sink consume energy faster in the existing routing algorithms, the authors propose a routing algorithm for dynamically adjusting the forward angle according to the residual energy. The algorithm introduces the residual energy and the nodal distance into an ant-colony probability transfer function to avoid premature death of critical nodes. In [17], the paper designs a routing algorithm depended on residual energy and communication cost between nodes. This algorithm takes full account of the remaining energy of all sensor nodes for cluster head selection and competition and selects the optimal node as the cluster head. Then a sensor with a small communication cost and a large amount of remaining energy will be used as the next hop to achieve energy balance. In [18], the authors propose a Clustering Protocol based on the Residual Energy Level (CPREL) that can effectively balance the energy dissipation of all network nodes and expand the network lifetime. In [19], the authors propose a type of energy-efficient clustering routing strategy based on Low-Energy Adaptive Clustering Hierarchy (LEACH). This strategy considers the level with two different nodes; namely, it selects the normal nodes with less energy as the cluster members and takes nodes with more remaining energy as the cluster head. At the same time, the strategy seeks the path to anchor with the smallest distance. To summarize, these algorithms take notice of the residual battery energy of nodes in the routing decision-making process and try to use high-energy nodes to forward data to extend the network lifetime. Meanwhile, the introduction of the distance factor can avoid excessive energy consumption and a long path of data sending nodes to a certain extent.

Here, there are also some strategic routing algorithms based on adjusting the node transmission distance to achieve the purpose of directly decreasing the node energy decay speed in [20-23]. Among them, a routing algorithm derived from a Nonlinear Weighted Particle Swarm Optimization algorithm (NWPSO) is introduced in [20] to prolong the network lifetime. By combining energy efficiency with transmission distance, the network can provide a better distributed and balanced sensor cluster system, thus improving the network life cycle. In [21], an ant colony routing algorithm has been developed; the algorithm uses the enhanced heuristic function and analyzes the distance of nodes, transmitting direction, and remaining energy, and it can work out the optimal route between source and destination. Considering the trade-off between the transmission radius and substantial transmission energy cost, the authors of [22] propose a WSN energy utilization model to achieve a higher packet of successful acceptance. To solve the energy hole problem, [23] proposes a dynamic routing algorithm 
based on a transmission distance adjustment method, which changes the nodes coverage with its residual energy and the distance to anchors, so that each node can attain energy balance.

Those methods roughly resolve the imbalance obstacle of energy consumption in WSNs from the perspective of the residual energy or node transmission distance. The implementation of energy balance routings in [24-28] is also an effective means of extending the life of the network. In [24], to minimize the total energy consumption minimize and equilibrate the network load, the authors put forward a General Self-organized Tree-based Energy Balance routing protocol (GSTEB). This protocol uses a process to build a routing tree and select a new root node in the process of each round. Then, each node selects its parent node depending on its neighbor's information. In [25], the authors propose a distributed routing algorithm based on game theory. The proposed protocol achieves a prolonged network lifetime by balancing communication loads across nodes in the region and within the region. In [26], to balance energy consumption, the authors designed a next-hop node selection algorithm based on a fuzzy classifier. It classifies nodes at relative levels with node's gradient. The maximum residual energy node at each level has the opportunity as the next hop. Then, in consideration of these algorithms and directed diffusion, this paper proposes an energy-balanced routing protocol to rationally utilize the limited energy, reduce the average energy consumption, and achieve WSN lifetime maximization. In [27], the authors model the monitoring region as a concentric centered on the sink and propose a mechanism to eliminate energy holes. This mechanism equalizes the energy loss of total nodes in the ring and extends the network lifetime. To effectively reduce and keep network energy balance, the authors in [28] analyzed different energy and flow models and proposed an Energy-balanced Distance-based Routing Algorithm (EDRA). The algorithm enables all nodes to utilize energy at a similar rate when the distance has been given.

It is one of the primary design objectives of WSNs to save and equalize the used energy and maximize the network survival time $[29,30]$. Important works from the literature, including the above-listed algorithms [13-28], provide some referential solutions. However, in a WSN, information congestion may occur when many sensors send data concurrently. In other words, congestion is more likely to happen when a sensor sends packets continuously. Because of this, the network packet loss ratio will increase, and the transmission efficiency of the network will be affected. Therefore, congestion control [31,32] becomes a key challenge. In [31], by taking advantage of the node's hop-by-hop feedback information mechanism, the Distributed Predictive Congestion Control algorithm (DPCC) is proposed. DPCC uses a queue utilization rate and an embedded channel estimation algorithm for congestion detection. Then the adaptive flow control of DPCC takes an adapted rate, so that the data stream can adaptively select the path of transmission, which partially alleviates network congestion. In [32], the authors develop a queuing network model to observe the congestion. They used the principle of the hydraulic flow and proposed a Congestion Control-based Optimal Routing algorithm (CCOR). For computing node location and packet service rate, CCOR introduces two functions: link gradient and traffic radius. Finally, the path selection probability is assigned in terms of the link flow. In [33], by designing the pipeline model under the spatial relationship and network load, the Traffic-aware and Energy-efficient Routing (TER) algorithm sends the packet to the sink, at the same time meeting energy efficiency and congestion avoidance as much as possible. In [34], the authors developed an Active Queue Management (AQM) algorithm. The algorithm combines random early detection with a fuzzy proportional-integral controller for congestion detection. If congestion occurs, the fuzzy logic alters the rate at which the node's packets are sent and the length of a buffer queue. Different from the energy routing algorithm, which aims at extending the network lifetime, the congestion control routing algorithm provides a guarantee for the success of data transmission and helps to improve the performance of the data-centric WSN.

It can be seen that for the routing design of WSNs, a large number of existing studies only consider one or several combinations of energy, distance, node queue, and other factors. It is undeniable that the algorithms proposed in this literature have indeed achieved some expected goals to some 
extent, but often, they degrade other aspects of network performance due to lack of consideration of other elements.

\subsection{Contributions}

With years of development, the routing protocol of WSNs has become an intense research field. However, as mentioned above, many studies have only proposed "special effect" algorithms; that is, some performance indicators are very prominent, while other aspects are difficult to compare with different algorithms. Therefore, based on the original intention of providing comprehensive and proper routing protocols, this paper designs a new algorithm termed multiparameter fusion decision routing (MPFDR). First, our algorithm establishes a multidimensional routing model, which lays a foundation for subsequent routing. Second, a new routing strategy based on contribution is proposed for the selection of the next hop. Furthermore, the main contributions of this paper are summarized as follows:

(1) We develop a routing evaluation model. Comprehensively integrating the residual energy and forward distance of neighbor node, single-hop transmission energy consumption, queue cache, energy equilibrium degree, and other indicators, we calculate the routing evaluation parameters of each forward neighbor.

(2) The routing selection algorithm based on the parameter contribution degree is proposed. The algorithm makes a concurrent contribution to enhancing the energy balance and effectiveness, alleviating node congestion, and improving transmission delay. According to the change of the relevant network state indicators of the current node and its forward neighbor region, this proposed strategy dynamically adjusts the reliability coefficient of each parameter to coordinate the decision influence force in the process of selecting the next hop. Moreover, after considering the parameter contribution degree of the forward neighbors and the fuzzy contribution degree, it calculates the fusion contribution degree of multiple scenes under the condition of ensuring at least one deterministic parameter contribution degree. Finally, the result of the superposition is used as the final routing decision criterion.

(3) In addition, we analyze the algorithm. Through theoretical analysis, the interval of the adjustment factor in the cache queue index and the influence of different values are determined. We provide the computational time complexity of our algorithm.

(4) Combined with the experimental simulation and comparison algorithms, we analyze the influence of the adjustment factor and network size on the network performance. The network performance metrics include the network lifetime, average energy consumption, packet loss ratio, and average hops. By contrast with the comparison algorithms, the MPFDR algorithm has the lowest packet loss ratio, which reflects good congestion control ability. Although the network lifetime is lower than that of MRE, and the average energy consumption and average hops are slightly higher than those of MPEC, other aspects of the proposed algorithm are better than those of the other two algorithms, and the overall performance has more obvious advantages.

The rest of the paper is organized as follows. Section 2 gives the system model and some relevant definitions. Section 3 describes the proposed routing optimization algorithm in detail and provides the implementation and analysis of our algorithm. Next, in Section 4, the effects of the adjustment factor and network size on performance are analyzed based on the simulation results and other algorithms. Finally, Section 5 summarizes the full text and future work.

\section{System Model and Routing Evaluation Parameter}

\subsection{System Model and Relevant Definitions}

The wireless sensor network considered in this paper contains $N$ isomorphic sensor nodes with the same initial state. Each node except the sink can receive and forward a packet and generate packets at a specific rate $r$. The sink node is responsible for aggregating and processing the data. Each node's maximum battery capacity is $E_{0}$, and the maximum communication radius is $R$. The sensor node will 
be dead if its battery energy is lower than 0 . The initial load value of all nodes is 0 , and their cache queue is a fixed value, so packets need to be processed in a first-in-first-out manner. In a square $S$, a sensor node is randomly deployed. The center of this square is the sink node. The entire sensor network forms an undirected graph $G(P, E)$. $P$ denotes the set of all nodes in the WSNs. $E$ denotes the set of edges. An edge $e_{i j} \in E$ implies that the data from node $i$ can be successfully received by node $j$; that is, node $j$ is located in the wireless signal coverage of node $i$.

The WSN involved in this article is primarily deployed in the data acquisition area. Senor nodes percept the surrounding environment or monitor particular objects. These nodes periodically produce and transmit data to the sink in wireless radio. To explain the MPFDR algorithm, we make the following definitions:

Definition 1. According to the wireless signal converge area, the neighbor set NB(i) of a node $i$ is expressed by the following:

$$
N B(i)=\left\{j \mid d_{i, j} \leq R, i, j \in P\right\}
$$

where $d_{i, j}$ is the geometrical distance between nodes $i$ and $j$.

Definition 2. Considering a packet located at node i, to transmit data against the sink without backhaul, the packet should be sent to a forward neighbor of node i. Based on the model in [35,36], the forward neighbor set $F N(i)$ of node $i$ can be expressed as follows:

$$
F N(i)=\left\{j \mid j \in N B(i), d_{j, \operatorname{sink}} \leq d_{i, \text { sink }}\right\}
$$

where $d_{i, \text { sink }}$ and $d_{j, \text { sink }}$ represent the distances from nodes $i$ or $j$ to sink. Here, the forward neighbor is one of the next hop candidates, which will be used hereafter.

Definition 3. $\Theta s i n k i$ is a circular region, and its center is the sink. It is radius is $d_{i, \text { sink }}$. $\Theta i$ in is another circular region with center node $i$ and radius $R$. Therefore, the forward neighbor area FNA $(i)$ of the sensor node $i$ is as follows:

$$
F N A(i)=\Theta \operatorname{sinki\cap } \Theta i \_R
$$

\subsection{Routing Evaluation Parameter}

In general, the design of the routing algorithm needs to consider node traffic load, residual energy, relative location, etc. In this paper, we introduce some routing evaluation parameters, including energy equilibrium degree, single-hop transmission ratio, effective forward rate, cache queue index, and residual energy level factor. Based on the five parameters, we establish a routing model.

Among the five parameters, the energy equilibrium degree reflects the degree of residual energy balance in the forward neighbor area, so that the node energy attenuation speed is kept as consistent as possible to prolong the network lifetime. Because of the data transmission capacity of the current node, the single-hop transmission distance is linked to its residual energy. Hence, a node with high residual energy can choose the forward neighbor with longer single-hop transmission distance. Conversely, those closer forward neighbors will be selected as much as possible. The effective forward rate reflects the location of the relationship between the nodes, which enable the packets to arrive at the sink as quickly as possible. The cache queue index and the residual energy level factor reflect the traffic load and residual energy of the forward neighbors, respectively, so that the packets in the transmission can avoid the congestion area and nodes with massive energy loss.

\subsubsection{Energy Equilibrium Degree}

In WSNs, the one-hop coverage area of adjacent nodes overlaps with each other. If local energy balance within the one-hop range can be achieved, the energy balance of the entire network can be 
approximately realized in a distributed way by using local information [37]. However, for the routing decision-making, some energy balance routing algorithms [16-19] generally consider the residual energy to select the next hop. In these algorithms, the node with less residual energy has a lower chance of being chosen as the transit node. At the same time, the nodes with more residual energy are more involved in data transmission. This literature takes residual energy as one of the criteria for routing decisions. In the traditional method, energy balance is passive; that is, the residual energy may have an extreme imbalance when the data forwarding path is updated. Therefore, our paper introduces an active energy balance strategy. Before data are formally transmitted, this strategy predicts the equilibrium degree of residual energy between nodes after selecting different neighbors.

In the traditional method, the energy imbalance in a local network region $A$ is measured by the mean square error of the residual energy of the nodes. Hence, the region energy imbalance can be expressed as follows:

$$
E I B^{\prime}=\sqrt{\frac{1}{n} \sum_{i \in A}\left(E_{i}-\bar{E}\right)^{2}}
$$

where $n$ is the node number of $A$. $E_{i}$ denotes the residual energy of node $i . \bar{E}$ represents the average residual energy of all sensor nodes. The mean square error cannot fully reflect the unbalance degree of the residual energy within $A$. For example, if the residual energy of each node is reduced by half, then $E I B^{\prime}$ is also reduced to half of the original. However, the energy imbalance between nodes does not change.

In sociology, the Atkinson coefficient is defined to measure the inequality of income distribution. The smaller the social income gap is, the lower the value of the Atkinson coefficient will be. Here, when the Atkinson coefficient is introduced into a WSN, the new EIB is as follows:

$$
E I B=1-\left[\frac{1}{n} \sum_{i \in A}\left(\frac{E_{i}}{\bar{E}}\right)^{1-\varepsilon}\right]^{\frac{1}{1-\varepsilon}}
$$

where $\varepsilon$ is the inequality aversion index, and the commonly used value is 2.5. EIB describes the energy balance state of nodes within the single-hop range; that is, if the energy distribution in $A$ is more balanced, the Atkinson index value will be smaller.

Thus, assuming that forward neighbor $h$ is the next hop of node $i$, the residual energy of node $i$ is as follows:

$$
\hat{E}_{i}^{i h}=E_{i}-e_{t}\left(l, d_{i, h}\right)
$$

where $l$ is the size of the unit packet. $d_{i, h}$ is the distance between node $i$ and $h . e_{t}\left(l, d_{i, h}\right)$ is the energy required to transmit $l$ bits data.

At the same time, the forward neighbor $h$ needs to receive and forward the data from node $i$. Therefore, its estimated residual energy can be expressed as follows:

$$
\hat{E}_{h}^{i h}=E_{h}-e_{r}(l)-e_{t}(l, R)
$$

where $e_{r}(l)$ and $e_{t}(l, R)$ represent the energy used to receive and send $l$ bits of data, respectively. Here, it is assumed that the distance forwarded by the forward neighbor $h$ is the maximum communication radius $R$.

For other forward neighbors, since they do not need to consume energy to participate in the data transmission of the node $i$, their remaining energy can be regarded as remaining unchanged, that is, the estimated remaining energy is as follows:

$$
\hat{E}_{j}^{i h}=E_{j}
$$


According to Equations (6) to (8), EIB of each candidate next hop in the set $F N(i)$ can be estimated. That is, after node $j$ is selected as the next hop and data transmission is performed, the imbalance of the remaining energy between the nodes in the radio radius of node $i$ is as follows:

$$
E I B^{i j}=1-\left[\frac{1}{n} \sum_{m \in F N(i)+\{i\}}\left(\frac{\hat{E}_{m}^{i j}}{\overline{\hat{E}}^{i j}}\right)^{1-\varepsilon \frac{1}{1-\varepsilon}}\right]
$$

where $\overline{\hat{E}}^{i j}$ represents the average residual energy of total nodes in the region, as follows:

$$
\overline{\hat{E}}^{i j}=\frac{1}{n} \sum_{m \in F N(i)+\{i\}} \hat{E}_{m}^{i j}
$$

Finally, when the current neighbor node $j$ is used as the next hop, the energy equilibrium degree between the all forward neighbors of a node $i$ is as follows:

$$
E B^{i j}=1-E I B^{i j}
$$

\subsubsection{Single-Hop Transmission Ratio}

Because of limited battery capacity, sensor nodes will eventually be exhausted. Therefore, when selecting the next hop, it should be clear how data transmission affects node energy. Generally, the energy consumption of a node includes three parts: perception, data processing, and communication. Of those, communication consumes much of the energy, more than the sum of perception and data processing consumption. Hence, this paper only considers the communication energy consumption. At the same time, because the energy consumption of data receiving is far less than that of transmitting, it can be neglected. Therefore, reducing the energy consumption of transmission between nodes is the key to slow down the energy attenuation speed and extend survival time. Thus, combining the residual energy of node $i$ and the transmission energy by single-hop, this paper defines the single-hop transmission ratio $E C^{i j}$, which is expressed as follows:

$$
E C^{i j}=E_{i} \times \frac{e_{R}-e_{i j}}{e_{R}}=E_{i} \times\left(1-\frac{e_{i j}}{e_{R}}\right)
$$

where $e_{i j}$ is the energy consumption of single-hop between node $i$ and $j . e_{R}$ is the energy consumption by transmitted to the maximum range. The communication energy consumption model used in this paper comes from $[35,36]$. Assuming that the communication distance threshold of sensor nodes is $d_{0}$, when $d$ is less than $d_{0}$, the free-space channel model is adopted. In contrast, the multichannel attenuation model will be taken. Then, the energy consumption for transmitting $l$ bits data is as follows:

$$
e_{t}(l, d)= \begin{cases}l E_{b a s}+l E_{f s} d^{2}, & d<d_{o} \\ l E_{b a s}+l E_{m p} d^{4}, & d \geq d_{o}\end{cases}
$$

The energy of receiving $l$ bits data is as follows:

$$
e_{r}(l)=l E_{b a s}
$$

where $E_{f s}$ and $E_{m p}$ are the amplification factor of the node radio signal amplifier. $E_{b a s}$ represents energy consumption per one bit of the control circuit in the node. 


\subsubsection{Effective Forward Rate}

Due to the limited coverage of sensor nodes, they rarely transfer data directly to the sink. Most nodes need forward data. Therefore, in the routing process, the geometry relationship in the current node, the forward neighbor, and the sink should also be considered.

For selecting the next hop, this paper introduces the effective forward rate $R_{f}^{i j}$. As shown in Figure $1, \Theta \operatorname{sink} j$ represents a circle with sink as the center and $d_{j, \operatorname{sink}}$ as the radius. A line isink represents a link between node $i$ and sink. Node $j^{\prime}$ is the intersection of circle $\Theta \sin k j$ and line isink. Here, the forward distance $d_{f}(i$,sink $)$ between node $i$ and the sink is defined as follows:

$$
d_{f}(i, \operatorname{sink})=d_{i, \sin k}
$$

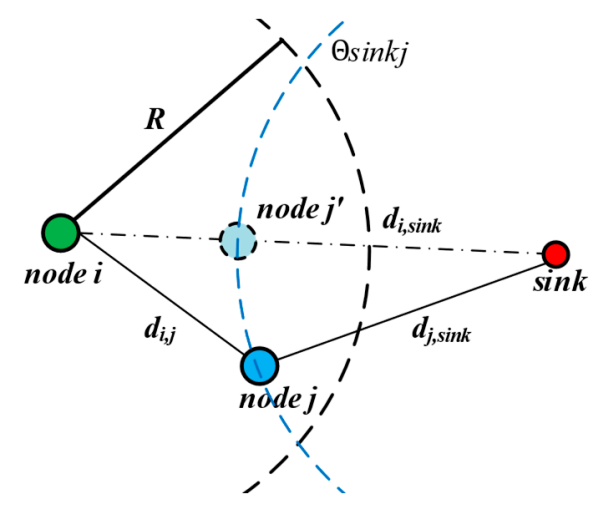

Figure 1. The forward distance.

Then, for a node $j \in F N(i)$, the forward distance from node $i$ to $j$ can be expressed as follows:

$$
d_{f}(i, j)=d_{i, j^{\prime}}=d_{i, \text { sink }}-d_{j, \text { sink }}, j \in F N(i)
$$

Finally, based on $d_{f}(i, j)$ and $d_{f}(i, \operatorname{sink})$, the effective forward rate $R_{f}^{i j}$ of the node $j$ can be expressed as follows:

$$
R_{f}^{i j}=\frac{d_{f}(i, j)}{d_{f}(i, \sin k)}
$$

The effective advancing rate considers the geometric distance relationship between nodes $i, j, \operatorname{sink}$; hence, it directs the packet flow directly to the sink. Thus, energy consumption can be cut down. In the free space model, sensor nodes tend to consider select neighbors with long single-hop transmission distance as the next hop. In this way, after the packets are forwarded to the next hop, they will be closer to the sink. Thus, the energy consumption of data transmission in a single-hop range is more productive and reduces the number of hops in the transmission path.

\subsubsection{Cache Queue Index}

Data transmission in WSNs based on static or event-driven data has distinct "many-to-one" characteristics; hence, in the case of traffic bursts, it is likely to cause network congestion and thus worsen the success rate of packet transmission. Since the cache occupancy of the sensor node is a reliable congestion detection, this property can be considered in the establishment of routing, which makes for the avoidance of those nodes with large cache occupancy and transfers the traffic load to idle or lightly loaded nodes. Finally, we can accomplish a fair utilization of the queue and improve network performance. Here, the cache occupancy rate of the next hop $j$ can be expressed as follows:

$$
r_{c}(j)=\frac{Q_{i}}{Q_{m}}
$$


where $Q_{j}$ is the current queue length of node $j . Q_{m}$ is the maximum queue length.

Cache occupancy $r_{c}(j)$ only reflects load for a single node. Although it is always better to select a low-occupancy cache node when choosing the next hop, this strategy does not take into account the impact of node cache on the load balance and energy balance of the overall network at different network sizes. Therefore, this article introduces an adjustment factor $\alpha$ and defines the cache queue index $\zeta$, taking into account the situation of the entire network. The adjustment factor $\alpha$ is set based on the size and performance requirements of WSN. The cache queue index $\zeta^{j}$ of node $j$ is defined as follows:

$$
\zeta^{j}=\sqrt{1-\left[r_{c}(j)\right]^{\alpha}}
$$

Because the $r_{c}(j)$ value interval is $[0,1], 1-\left[r_{c}(j)\right]^{\alpha}$ causes the result to be non-negative. The square root operation makes that $\zeta^{j}$ has a nonlinear relationship with $r_{c}(j)$. When $r_{c}(j)$ is smaller, $\zeta^{j}$ is higher, that is, node $j$ has more cache and should forward a large amount of data; conversely, when $r_{c}(j)$ is close to 1 , node $j$ 's cache is approximately full. It almost should not forward to any packet to avoid overload. Therefore, the nodes with the higher cache queue index should be selected as much as possible to reduce the packet loss ratio and ensure the success of data collection. In Sections 3.3.1 and 4.1, we make a detailed analysis of the relationship between the value of the adjustment factor $\alpha$ and the network size and performance.

\subsubsection{Residual Energy Level Factor}

In general, node energy is always a vital index for designing and evaluating routing algorithm in wireless sensor networks. For those parameters of this paper defined above, to take care of the energy consumption of the current node $i$, we have considered the residual energy of node $i$, single-hop data transmission distance $d_{i, j}$, and forward distance $d_{i, j^{\prime}}$. However, it also should not happen that the routing ignores the residual energy state of the forward neighbor $j$, and it is necessary to introduce the residual energy level as a reference factor. Here, the residual energy level factor $R E^{i j}$ of the forward neighbor $j$ is defined as follows:

$$
R E^{i j}=\frac{E_{j}}{E_{o}}
$$

where $E_{j}$ is the residual energy of forwarding neighbor $j . E_{o}$ is the initial node energy.

\section{Proposed Routing Optimization Algorithm}

\subsection{Principle and Implementation}

For a given WSN, to comprehensively consider the impact of the routing evaluation parameters defined in Section 2.2, we proposed a routing optimization algorithm based on a multiparameter fusion decision, termed the MPFDR algorithm. The basic principle of the algorithm is to construct a transmission link to the next hop with the maximum fusion contribution degree. In other words, if node $i$ wants to send data, the next hop will be selected from the forward neighbors according to the defined routing optimization based on parameter fusion strategy. Simultaneously, if the sink is within the one-hop range of node $i$, data can also be sent to it directly.

Implementation of our MPFDR algorithm mainly includes three processes: establishing the forward neighbor information table, calculating routing evaluation parameters, and selecting the next-hop node from the forward neighbor set.

\subsubsection{Establishment of the Forward Neighbor Information Table}

In the initial phase, the sink sends an initialization message to all of the nodes in the maximum power. After receiving this message, every node evaluates a similar distance to the sink, depending on the Received Signal Strength Indicator (RSSI). Then, the node sends the neighbor discovery message to nodes within the one-hop range in the default transmission power. The message consists of the node's 
identification, the distance to sink, the current residual energy, the cache queue size, etc. The node that received this message will measure the distance to its neighbor and establish forward neighbor set $F N(i)$ according to Equations (1) and (2). Hence, the forward neighbor information table can be created.

\subsubsection{Calculation of the Routing Evaluation Parameters}

Based on the forward neighbor information table, the routing evaluation parameters of total forward neighbors, including the energy equilibrium degree, single-hop transmission ratio, effective forward rate, cache queue index, and residual energy level factor, can be calculated according to the routing model of Section 2. In the process, node $i$ gathers the relevant information about the forward neighbor $j$ for subsequent routing selection. This information includes its residual energy, the distance to the forward neighbor $j$ and the sink, the residual energy and the queue length of node $j$, the distance between node $j$ and the sink, and so on.

\subsubsection{Selection of the Next Hop}

Based on the determined routing optimization parameters, the fusion contribution degree of each forward neighbor is calculated according to the routing selection strategy in Section 3.2. When the sensor node detects or forwards data, the next hop $g$ is the node that has the maximum fusion contribution degree among the forward neighbor set $F N(i)$.

\subsection{Routing Selection Strategy}

Regarding total forward neighbor nodes of the current node $i$, the higher the numerical difference in the routing evaluation parameter $k(1 \leq k \leq K)$, the higher its importance on the next-hop selection and the higher the reliability. In contrast, it indicates that it plays a limited role in the decision-making of the next hop, that is, the reliability is low. Therefore, in order to represent this characteristic of routing parameters, the reliability coefficient $\wp^{k}$ is introduced here, and its specific calculation method is as follows:

First, the $k^{\text {th }}$ evaluation parameter of the candidate next hop $j$ is $v_{j k}$, and the initial matrix $D=\left\{v_{j k}\right\}$ is established accordingly. Thus, the standard deviation $\sigma_{k}$ of each parameter can be calculated as follows:

$$
\sigma_{k}=\sqrt{\frac{\sum_{j \in F N(i)}\left(v_{j k}-\bar{v}_{k}\right)^{2}}{|F N(i)|-1}}
$$

where $\bar{v}_{k}$ represents the mean of the $k^{\text {th }}$ parameter value of all forward neighbors and $|F N(i)|$ is the modulus of the forward neighbor set, that is, the number of candidate next hops. Then, the reliability coefficient $\wp_{k}$ of the parameter $k$ is as follows:

$$
\wp_{k}=\frac{\bar{v}_{k} \cdot \sigma_{k}}{\sum_{k=1}^{K}\left(\bar{v}_{k} \cdot \sigma_{k}\right)}
$$

where $K$ is the number of routing evaluation parameters. Here, considering that $\sigma_{k}$ maybe close to or even equal to each other, to distinguish different optimization parameters, $\bar{v}_{k}$ is added to the calculation of the reliability coefficient.

Furthermore, for the forward neighbor $j$, its value reflected in each parameter $k$ contributes to its selection as the next hop. Therefore, the parameter contribution degree $m_{j}(k)$, as shown in the following equation, is defined in this paper, namely,

$$
m_{j}(k)=\frac{v_{j k}}{\left[\sum_{j=1}^{|F N(i)|} v_{j k}+K\left(1-\wp_{k}\right)\right]}
$$


Here, different from the general way of assigning the contribution degree only according to the proportion of the parameter value, this paper introduces a modified value $K\left(1-\wp_{k}\right)$ into the denominator of $m_{j}(k)$, so that the greater the reliability coefficient of parameter $k$ is, the greater the parameter contribution degree of forward neighbor $j$, which is selected as the next hop, and vice versa. Therefore, the reliability degree of routing parameters is well integrated into the calculation of the contribution degree, so that the parameter value not only reflects the node state but can also feedback the network state fluctuation of the forward neighbor area on the routing optimization parameters.

Generally, when the parameter contribution degree of all routing evaluation parameters of the forward neighbor node $j$ is obtained, there are two methods for selecting the next hop. One is to choose the node with the maximum contribution degree as the next hop. The other needs to compute a weighted sum of evaluation parameters for each node, then select the maximum sum node as the next hop. However, the former method makes a decision only based on the contribution degree, ignores the other necessary settings, which can also reflect fluctuations in the network status. Meanwhile, the second method may have a more significant deviation due to manually determining the routing parameter weights. Therefore, to avoid a too one-sided selection of the next hop and eliminate subjective factors as much as possible, this paper proposes a multiparameter fusion strategy. In the process of routing, for the sake of setting an appropriate selection of the next hop from $F N(i)$, this strategy integrates the parameter contribution degree $m_{j}(k)$ of forward neighbor $j$ and the fuzzy contribution degree $m_{\Omega}(k)$ and calculates the fusion contribution degree as the final standard of routing decisions.

From the existence of different reliability coefficients for each route evaluation parameter, the parameter itself, as the deciding factor for selecting the next hop, has some indeterminacy $\Omega$, which is derived from the suitability of the evaluation parameter as one of the routing decision factors. Therefore, to characterize it, the fuzzy contribution degree of parameter $k$ about indeterminacy $\Omega$ is defined as follows:

$$
m_{\Omega}(k)=1-\sum_{j=1}^{|F N(i)|} m_{j}(k)
$$

By Equations (23) and (24), the parameter $k$ contribution degree $m_{j}(k)$ of forward neighbor node $j$ and the fuzzy contribution degree $m_{\Omega}(k)$ of indeterminacy $\Omega$ to $k$ can be respectively calculated. Concurrently, although there are uncertainties in the routing evaluation parameters, both $m_{j}(k)$ and $m_{\Omega}(k)$ are direct manifestations of the network state of the forward neighbor area $F N A(i)$ on a parameter $k$; therefore, any simple human abnegation of them may lose important information (e.g., threshold value method), and the choice of the next-hop node is flawed. Therefore, to more clearly explain and implement the routing optimization strategy in this paper, the following calculation method of fusion contribution degree $m_{j}(V)$ is defined.

Here, considering that not all routing evaluation parameters are in a state of indeterminacy, it should be ensured that the calculation of the contribution degree under each scenario combination includes at least one parameter contribution degree. Thus, a one-dimensional set $\theta=\left\{\theta_{1}, \theta_{2}, \cdots, \theta_{\eta}, \cdots, \theta_{T}\right\}$ is defined and $\theta_{\eta}$ is a random binary sequence of length $K$ except for all zeros, representing a scene combination. By traversing all the $T=2^{K}-1$ binary sequence combinations, the fusion contribution degree $m_{j}(V)$ of the forward neighbor can be obtained, and the formula can be expressed as follows:

$$
m_{j}(V)=\sum_{\eta=1}^{T}\left\langle\prod_{k=1}^{K}\left\{\left[m_{j}(k)\right]^{\theta_{\eta}^{k}} \cdot\left[m_{\Omega}(k)\right]^{1-\theta_{\eta}^{k}}\right\}\right\rangle
$$

where $\theta_{\eta}^{k}$ is the $k^{\text {th }}$ value of the binary sequence $\theta_{\eta}$, corresponding to the parameter $k$. When $\theta_{\eta}^{k}$ it is equal to $1, k$ takes the parameter contribution degree. When $\theta_{\eta}^{k}$ it is equal to $0, k$ takes the fuzzy contribution degree. 
Finally, in $F N A(i)$, the current node $i$ selects the forward neighbor with the maximum fusion contribution degree as the next hop $g$, that is,

$$
g=\max _{j}\left[m_{j}(V)\right], j \in F N(i)
$$

\subsection{Performance Analysis}

\subsubsection{Influence of the Adjustment Factor on the Network Performance}

In this section, for the proposed cache queue index, it is significant to decide the value interval of the adjustment factor $\alpha$ and the influence of different values on the relevant network performance. The definition of the cache queue index is shown as Equation (19). For a theoretical analysis, we alter a variable name. The cache occupancy rate $r_{c}(j)$ is replaced with $x$. The cache queue index $\zeta^{j}$ is replaced with $f(x)$. The new equation of the cache queue index can be expressed as follows:

$$
f(x)=\sqrt{1-x^{\alpha}}, x \in[0,1]
$$

(1) First, to make $f(x)$ meaningful, the following should be true: $1-x^{\alpha} \geq 0$. Considering this, the cache occupancy rate should satisfy $x \in[0,1]$, and $\alpha$ is not equal to 0 , but $\alpha>0$.

(2) When $0<\alpha<1$, the derivative of $f(x)$ is:

$$
f^{\prime}(x)= \begin{cases}-\infty, & x=0 \text { or } 1 \\ \frac{-\alpha x^{\alpha-1}}{2 \sqrt{1-x^{\alpha}}}, & 0<x<1\end{cases}
$$

By setting $\psi(x)=\frac{x^{\alpha-1}}{\sqrt{1-x^{\alpha}}}$ and taking its derivative, we have the following:

$$
\psi^{\prime}(x)=\left(1-x^{\alpha}\right)^{-\frac{1}{2}} \cdot\left[(1-\alpha) x^{-\alpha}+\frac{\alpha}{2}-1\right]
$$

From the above equation, we know that there exists $\psi^{\prime}(x)>0$ when $x \in(0, \sqrt[\alpha]{(2-2 \alpha) /(2-\alpha)})$, and it is $\psi^{\prime}(x)<0$ when $x \in(\sqrt[\alpha]{(2-2 \alpha) /(2-\alpha)}, 1)$. Therefore, $f^{\prime}(x)$ is always less than 0 , and it increases first and then decreases as $x$ increases. Under this adjustment factor interval, when $x$ is in the front part of the interval, that is, the cache occupancy rate is low, the corresponding cache queue index sharply decreases with the increase of the cache occupation rate. At this point, due to the "overreaction" of the cache queue index, the resources of the node cannot be effectively utilized, which may harm the network performance. In the meantime, as $\alpha$ approaches 1 , due to $\sqrt[\alpha]{(2-2 \alpha) /(2-\alpha)}$ decreasing until it reaches 0 , the proportion of the front part to the entire independent variable interval continues to decrease until it reaches 0 ; thereby, the network performance will also be improved.

(3) When $\alpha \geq 1$, the derivative of $f(x)$ can be similarly obtained as follows:

$$
f^{\prime}(x)= \begin{cases}0, & x=0 \\ -, & x<1 \\ -\infty, & x=1\end{cases}
$$

At this time, $\psi^{\prime}(x) \leq 0$ always holds, and $\psi^{\prime}(x)$ decreases with increasing $x$, that is, the cache queue index decreases as the cache occupancy rate increases. As $\alpha$ increases, the early-stage increment of the reduction rate gradually decreases, while the late-stage increment gradually increases. It is foreseeable that if $\alpha$ is too large, the "lazy reaction" of the cache queue index fails to reflect the degree of congestion of the node on time, which will lead to deterioration of the network performance.

In conclusion, the value interval of $\alpha$ is $(0, \infty)$. In this interval, the cache queue index can alleviate the node congestion, reduce the node energy burden, and improve the packet loss ratio. Meanwhile, 
when $\alpha$ is in $(0,1)$ or $[1, \infty)$, both the node congestion and energy balance effect will be developed as its value approaches 1 .

\subsubsection{Simple Estimate of the Computational Time Complexity}

The MPFDR algorithm primarily selects the next hop from nodes in the forward neighbor area, and the nodes in the area are fixed after the WSN is deployed. Each forward neighbor node calculates the five routing evaluation parameters defined by this algorithm, respectively. Hence, the calculation of these evaluation parameters is distributed parallel. When routing-decisions are made, the node calculates the parameter contribution degree and fuzzy contribution degree of each forward neighbor node. The two values are linearly related to the number of nodes $N$; hence, their time complexities both are $O(N)$. Then the fusion contribution degree is also a linear correlation with $N$; its time complexity is $O(N)$. Finally, all forward neighbor node's fusion contribution degrees are sorted by the merge sort algorithm to attain the maximum. The algorithm's time complexity is $O(N \log N)$. To summarize, the time complexity of the MPFDR algorithm is $O(N \log N)$.

\section{Simulation Results and Analysis}

For inspecting the efficacy and reliability of the MPFDR algorithm, simulations were conducted using MATLAB 2018b on a machine running Windows 10. The machine has an Intel Xeon E5-2620 v4 CPU and 32GB memory. The parameters setup for the simulation of the proposed algorithm is reported in Table 1. In this section, a comprehensive comparison is carried out based on the simulation results. In terms of performance analysis, the following three types of comparative performance indicators are adopted: the network lifetime (NL), average hops $(\mathrm{AH})$, average energy $(\mathrm{AE})$, and packet loss ratio (PLR). Among them, as mentioned in definition 4, NL can show the effect of the algorithm in balancing node energy to maximize the WSN lifetime. PLR may be evaluated as the ratio of packets that should have been received by the sink but were not. It can effectively indicate transmission reliability. AH can indicate the network delay. It may be measured as the number of nodes in which a packet passes from a source node to a sink. AE denotes the average energy consumption of the entire network in every transmission period, which can measure the energy economy.

Table 1. Simulation Parameters Values.

\begin{tabular}{ccc}
\hline Parameter & Value & Note \\
\hline Network coverage & $100 \mathrm{~m} \times 100 \mathrm{~m}$ & $\mathrm{~S}$ \\
Node number & $100 \sim 300$ nodes & $N$ \\
Battery initial energy & $0.5 \mathrm{~J}$ & $E o$ \\
Maximum communication radius & $30 \mathrm{~m}$ & $R$ \\
Packet generation rate & One packet/round & $r$ \\
Packet size & 1024 bits & $d m$ \\
Buffer size & 20 packets & $Q m$ \\
Adjustment factor & $0 \sim 20$ & $\alpha$ \\
Inequality aversion index & 2.5 & $\varepsilon$ \\
\hline
\end{tabular}

\subsection{Network Performance with Different Adjustment Factors}

As shown in Figures 2 and 3 under different network sizes with a step size of 50, the network performance index changes with the adjustment factor. It can be seen that in the process of selecting the next hop, the situation that some nodes are overused is somewhat relieved due to the influence of the cache queue index of high-cache-usage nodes. Thus, the adjustment factor $\alpha$ has more noticeable effects on NL and PLR, while impacts on AE and AH are relatively limited. When $\alpha$ is in $(0,1), \mathrm{NL}$ and PLR are improved with its increase. In contrast, when it is in $[1,20]$, both of them will increase $\alpha$. Here, it needs to be explained in combination with the analysis in Section 3.3.1. 


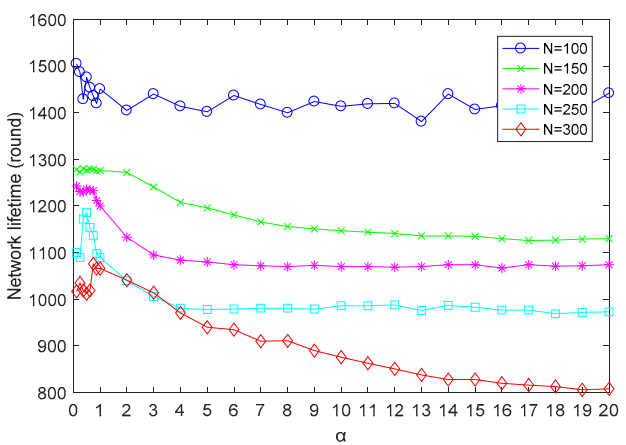

(a)

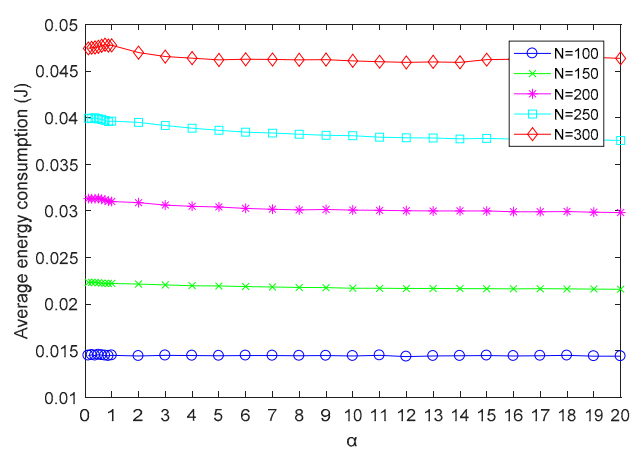

(b)

Figure 2. (a) Impacts of $\alpha$ on network lifetime (NL); (b) Impacts of $\alpha$ on average energy (AE).

When $\alpha \geq 1$, as $\alpha$ increases, NL gradually decreases, and the packet loss ratio also increases. Its reason is that with the rise in $\alpha$, the "punishment intensity" increases at first, while it is just the opposite in the later period. Therefore, when $\alpha$ increases to a certain extent, the routing interferes with node congestion during its early and middle stages, thus failing to prevent those nodes in high forwarding task state from being repeatedly selected on time; therefore, the network performance starts to decline.

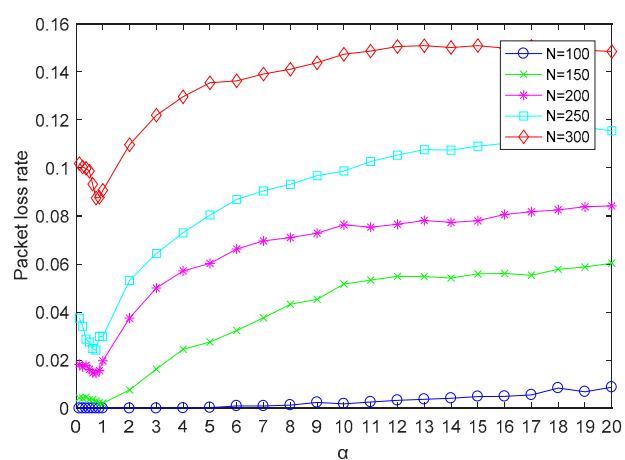

(a)

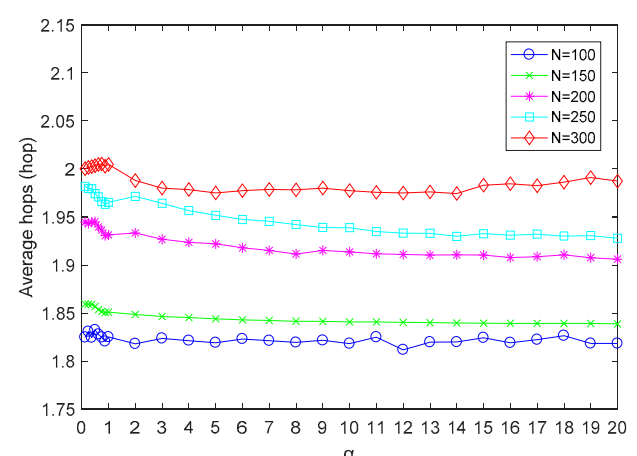

(b)

Figure 3. (a) Impacts of $\alpha$ on packet loss rate (PLR); (b) Impacts of $\alpha$ on average hops (AH).

From the above analysis, different $\alpha$ values will bring about fluctuations in network performance. If the network size is small, $\alpha<1$ will have little impact. If the network size is greater than $250, \alpha<1$ will cause the number of $\mathrm{AHs}$ to increase. If focusing on lower $\mathrm{AH}$ and $\mathrm{AE}, \alpha$ should take a larger value, but $\alpha>5$ will increase the PLR and decrease the NL. If concerned with packet loss and lifetime, $\alpha$ should take values between 0 and 1 . Considering all things, in the subsequent simulation, we take the adjustment factor $\alpha=1.3$.

\subsection{Comparison Algorithms}

In this section, three classical routing algorithms, MRE, MTEC, and MPEC, as well as two novel algorithms, the Energy Cost-Based energy Routing algorithm (ECBR) [38] and the Energy-Balanced Routing Protocol based on Forward-Aware Factor (FAF-EBRP) [39], are compared with our proposed algorithm. Moreover, we analyze the impact of the node number on NL, AE, PLR, and AH, and the results prove that the MPFDR algorithm enhances network lifetime. In Figures 4 and 5, NL, AE, PLR, and $\mathrm{AH}$ under different numbers of nodes are given. It can be seen that the MPFDR algorithm has the lowest PLR and outperforms the other algorithms in congestion control capability. Meanwhile, although NL is lower than that of MRE, and AE and AH are slightly higher than those of MPEC, the other performance indicators of the MPFDR algorithm are superior to those of the two algorithms. Therefore, the proposed MPFDR algorithm has apparent advantages. 


\subsubsection{Classical Algorithm: Network Performance with the Numbers of Nodes}

The MPEC algorithm chooses a path for data transmission from source to sink. Nodes in this path consume minimum energy but have a more massive load. Hence, MPEC 's NL is the shortest because of the higher packet loss ratio and easy premature failure. At the same time, due to the minimum energy consumption path being also the shortest, MPEC has the lowest AH. The MTEC algorithm selects the next hop with the shortest single-hop distance in the forward neighbor set. It is a selfish algorithm with "self-interested behavior", that is, it only considers the energy of the current node in the routing process, but ignores the remaining energy and cache occupation of the next hop. Therefore, in the end, the NL and PLR values of MTEC are not ideal. For AE and AH, because the single-hop distance is the shortest each time, the final path is almost the longest compared with other algorithms, resulting in higher $\mathrm{AE}$ and $\mathrm{AH}$. The MRE algorithm takes the residual energy as the routing standard and selects the high-energy node to forward data depending on the node energy change as far as possible. Therefore, the NL of this algorithm is the longest. However, since MRE chooses the node with the most residual energy to forward data, its routing path is usually long, resulting in the maximum $\mathrm{AE}$ of the network, and the AH is second only to MTEC.

In conclusion, due to the one-sided consideration, it is difficult for the three classical comparison algorithms to compare with other algorithms in some partial performance. In contrast, this paper considers the algorithm from various aspects and selects the optimal forward neighbor as the forwarding node according to the parameter fusion strategy. Therefore, as we can observe from the simulation results, the performance of MPEC in terms of AE and AH is slightly inferior to that of MPEC, the comprehensive property of the algorithm in this paper has reached better results when it compares with the four performance indicators.

\subsubsection{Recent Algorithm: Network Performance with the Numbers of Nodes}

From the perspective of the forwarding region of the next hop, the FAF-EBRP algorithm considers the ratio of the forward energy density of forwarding neighbors in all candidate next hops. Both the large number and the excellent energy condition of optional next hops can make the forward energy density relatively large; therefore, a large ratio indicates that it is more capable of undertaking the task of data forwarding. Second, for the quality of the forwarding communication link, the ratio of the forwarding link weight in all the alternative links is considered.

The ECBR algorithm evaluates minimum energy consumption to find a path to the sink. This algorithm defines the energy cost with single-hop transmission energy and the node residual energy. The forward neighbor $j$ is the forwarding node because it has the minimum sum of the energy cost from node $i$ to it and the energy cost from it to the next hop $h$.

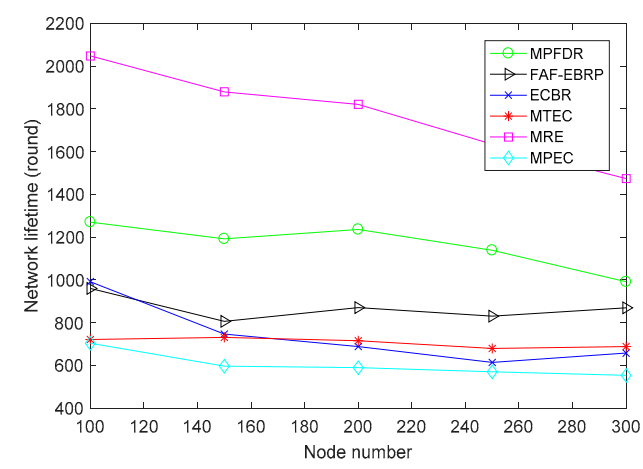

(a)

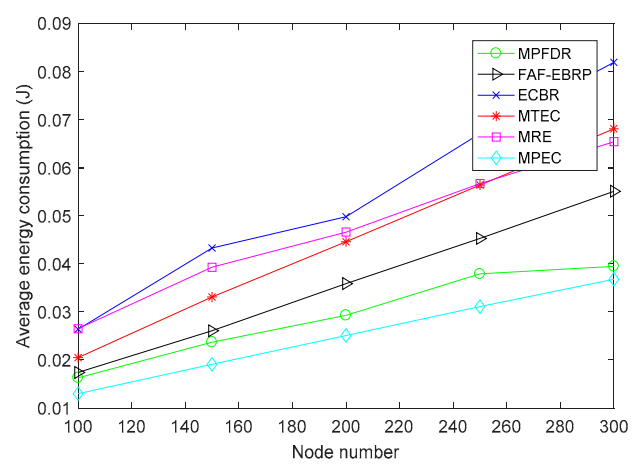

(b)

Figure 4. (a) NL versus different number of nodes; (b) AE versus different number of nodes.

In summary, in addition to considering the residual energy, the ECBR algorithm considers the energy of transferring data to the candidate node. The FAF-EBRP algorithm percepts link quality 
and energy density and considers various factors comprehensively; therefore, it is more successful in balancing energy consumption and shortening the transmission distance. Hence, FAF-EBRP is more advantageous than ECBR in terms of NL, AE, and AH, but not PLR. Of course, the MPFDR algorithm comprehensively considers the remaining energy and forward distance, single-hop transmission energy consumption, queue cache, energy balance, and other indicators to calculate routing optimization parameters. Concurrently, combined with the defined routing strategy, the reliability coefficient of each parameter is dynamically adjusted according to the change of related indicators of the current node and its forward neighbor area. In other words, because each parameter can reflect a different state of the forward neighbor node, our algorithm can coordinate its own decision-making influence according to parameters. Hence, it provides the most effective routing algorithm to improve energy balance, mitigate node congestion, and improve transmission delay. The above results prove that the proposed MPFDR algorithm significantly outperforms FAF-EBRP and ECBR in all four types of network performance indicators.

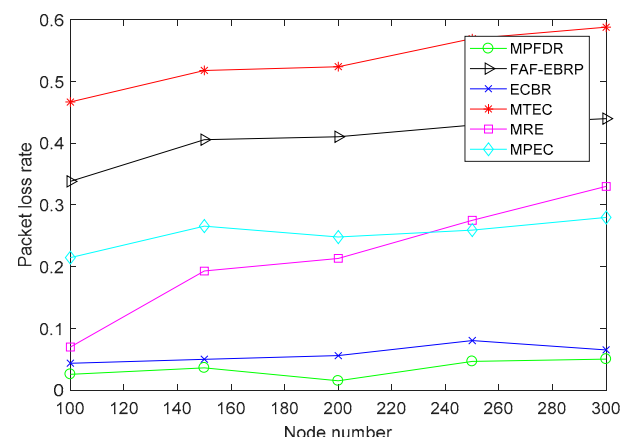

(a)

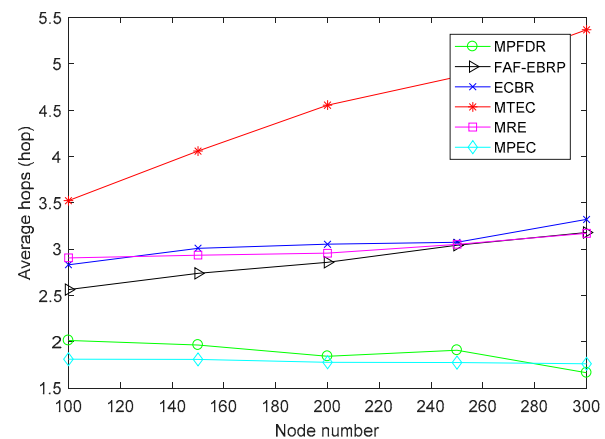

(b)

Figure 5. (a) PLR versus different number of nodes; (b) AH versus different number of nodes.

\section{Conclusions}

In this paper, we focused on routing optimization based on the multiparameter fusion decision on the energy-constrained wireless sensor networks. The MPFDR algorithm comprehensively considers the residual energy, forward distance, single-hop transmission energy, buffer queue, and energy equilibrium degree for choosing the optimum next hop; hence, the algorithm can realize a multidirectional reflection of the network state. Meanwhile, combined with the defined routing strategy, the fusion contribution degree of each forward neighbor is obtained. Then the node with the maximum fusion contribution degree will be selected as the next hop.

The simulation results show that our algorithm has better congestion control ability; that is, there are a lower packet loss rate and a lower transmission delay (average hops). The latency does not change dramatically with the increase of network size. Moreover, while ensuring low latency, the average energy consumption is adequately controlled. The result also shows that the MPFDR algorithm is more suitable for delay-sensitive applications, for example, substation environmental monitoring or medical equipment monitoring. In future research, we will focus on the application of the algorithm, mainly with the implementation of WSNs in power monitoring and industry 4.0 environments. For this, we will design a validation scheme, including three parts: (1) we will develop the WSN node based on the ZigBee protocol. (2) Scene 1: we randomly deploy nodes in a flat outdoor region, with a sink node located in the center, to verify performance in the line-of-sight environment. (3) Scene 2: we will deploy nodes in the building region to verify performance in a non-line-of-sight and object shielding environment. Through the scheme, we will attain our algorithm performance data in a real scenario and further analyze and improve the algorithm. In the future, we will study how to reduce transmission delays and improve transmission reliability, to meet the requirements of power accuracy control. 
Author Contributions: Conceptualization, J.Y.; methodology, J.Y. and L.T.; software, J.C. and Z.L.; validation, J.Y.; formal analysis, J.Y., J.C. and L.T; investigation, J.Y.; resources, R.W. and L.T.; data curation, Z.L. and J.C.; writing — original draft preparation, J.Y. and J.C.; writing—review and editing, J.Y. and L.T.; visualization, J.C.; supervision, R.W. and L.T.; funding acquisition, J.Y. All authors have read and agreed to the published version of the manuscript.

Funding: This work was supported by the Fundamental Research Funds for the Central Universities 2016 MS45.

Conflicts of Interest: The authors declare no conflict of interest.

\section{References}

1. Csáji, B.C.; Kemény, Z.; Pedone, G.; Andras, K.; Váncza, J. Wireless Multi-Sensor Networks for Smart Cities: A Prototype System with Statistical Data Analysis. IEEE Sens. J. 2017, 17, 7667-7676. [CrossRef]

2. Li, M.; Lin, H.J. Design and Implementation of Smart Home Control Systems Based on Wireless Sensor Networks and Power Line Communications. IEEE Trans. Ind. Electron. 2015, 62, 4430-4442. [CrossRef]

3. Yuan, Y.K.; Zhang, Y.; Wei, T.Y.; Yang, M.L.; Tan, Q.L. Review of key technologies and applications of intelligent transportation. Appl. Electron. Tech. 2015, 41, 9-12, 16. [CrossRef]

4. Nafi, N.S.; Ahmed, K.; Datta, M.; Gregory, M.A. A novel software defined wireless sensor network based grid to vehicle load management system. In Proceedings of the 2016 10th International Conference on Signal Processing and Communication Systems (ICSPCS), Gold Coast, QLD, Australia, 19-21 December 2016; pp. 1-6. [CrossRef]

5. Hamouda, Y.E.M.; Msallam, M.M. Smart heterogeneous precision agriculture using wireless sensor network based on extended Kalman filter. Neural Comput. Appl. 2018, 1-17. [CrossRef]

6. Moribe, T.; Okada, H.; Kobayashl, K.; Katayama, M. Combination of a wireless sensor network and drone using infrared thermometers for smart agriculture. In Proceedings of the 15th IEEE Annual Consumer Communications and Networking Conference (CCNC), Las Vegas, NV, USA, 12-15 January 2018; pp. 1-5. [CrossRef]

7. De, P.A.; Filho, R.H.; Rodrigues, J.; Oliveira, P. Infrastructure for Integration of Legacy Electrical Equipment into a Smart-Grid Using Wireless Sensor Networks. Sensors 2018, 18, 1312. [CrossRef]

8. Queiroz, D.V.; Alencar, M.S.; Gomes, R.D.; Fonseca, I.E.; Benavente-Peces, C. Survey and Systematic Mapping of Industrial Wireless Sensor Networks. J. Netw. Comput. Appl. 2017, 97, 96-125. [CrossRef]

9. Kassan, R.; Châtelet, E.; Soukieh, J. Reliability assessment of photovoltaic wireless sensor networks for forest fire propagation detection. Int. J. Model. Simul. 2018, 38, 50-65. [CrossRef]

10. Sinulingga, E.; Siregar, B. Remote Monitoring of Post-eruption Volcano Environment Based-On Wireless Sensor Network (WSN): The Mount Sinabung Case. J. Phys. Conf. Ser. 2017, 801, 012084. [CrossRef]

11. Lara, R.; Benítez, D.; Caamaño, A.; Zennaro, M.; Rojo-Álvarez, Z. On Real-Time Performance Evaluation of Volcano-Monitoring Systems with Wireless Sensor Networks. IEEE Sens. J. 2015, 15, 3514-3523. [CrossRef]

12. Roy, S.; Nene, M.J. A security framework for military application on infrastructure based wireless sensor network. In Proceedings of the IEEE International Conference on Research in Computational Intelligence and Communication Networks (ICRCICN), Kolkata, West Bengal, India, 20-22 November 2015; pp. 369-376. [CrossRef]

13. Han, K.H.; Ko, Y.B.; Kim, J.H. A novel gradient approach for efficient data dissemination in wireless sensor networks. In Proceedings of the IEEE 60th Vehicular Technology Conference, Los Angeles, CA, USA, $26-29$ September 2004; pp. 2979-2983. [CrossRef]

14. Singh, S.; Woo, M.; Raghavendra, C.S. Power-aware Routing in Mobile Ad Hoc Networks. In Proceedings of the 4th Nnnual ACM/IEEE International Conference on Mobile Computing and Networking, Dallas, TX, USA, 25-30 October 1998; pp. 181-190. [CrossRef]

15. Weng, C.C.; Chen, C.W.; Chen, P.Y.; Chang, K.C. Design of an energy-efficient cross-layer protocol for mobile ad hoc networks. IET Commun. 2013, 7, 217-228. [CrossRef]

16. Zhang, M.X.; Wang, H.F.; Xiang, F.H.; Mao, J.L.; Zhang, C.L. Routing algorithm on dynamic adjustment of forward angle based on residual energy. J. Comput. Appl. 2016, 36, 77-80, 86.

17. Tian, Y.F.; Wang, L.H. Routing algorithm for wireless sensor networks by considering residual energy and communication cost. J. Nanjing Univ. Sci. Technol. 2018, 42, 96-101. [CrossRef] 
18. Yuan, X.; Zhong, F.M.; Chen, Z.K.; Yang, D.L. Residual energy level based clustering routing protocol for wireless sensor networks. In Proceedings of the 6th International Conference on Electronics and Information Engineering, Dalian, China, 3 December 2015; p. 97940K. [CrossRef]

19. Singh, J.; Singh, B.P.; Shaw, S. A new LEACH-based routing protocol for energy optimization in wireless sensor network. In Proceedings of the International Conference on Computer and Communication Technology (ICCCT), Allahabad, India, 26-28 September 2014; pp. 181-186. [CrossRef]

20. Wang, N.; Zhou, Y.; Liu, J. An efficient routing algorithm to prolong network lifetime in wireless sensor networks. In Proceedings of the 10th International Conference on Communications and Networking in China (ChinaCom), Shanghai, China, 15-17 August 2015; pp. 322-325. [CrossRef]

21. Sun, Y.; Dong, W.; Chen, Y. An Improved Routing Algorithm Based on Ant Colony Optimization in Wireless Sensor Networks. IEEE Commun. Lett. 2017, 21, 1317-1320. [CrossRef]

22. Zhang, L.; Lim, A. Improving Lifetime in Maximum Residual Energy Routing With Increased Transmission Distance and Retransmission. In Proceedings of the International Conference on Communications, Circuits and Systems, Guilin, China, 25-28 June 2006; pp. 1438-1442. [CrossRef]

23. Tranquang, V.; Miyoshi, T. A transmission range adjustment algorithm to avoid energy holes in wireless sensor networks. In Proceedings of the 8th Asia-Pacific Symposium on Information and Telecommunication Technologies, Kuching, Malaysia, 15-18 June 2010; pp. 1-6.

24. Han, Z.; Wu, J.; Zhang, J.; Liu, L.; Tian, K. A General Self-Organized Tree-Based Energy-Balance Routing Protocol for Wireless Sensor Network. IEEE Trans. Nucl. Sci. 2014, 61, 732-740. [CrossRef]

25. Abd, M.A.; Al-Rubeaai, S.F.M.; Singh, B.K.; Tepe, K.E.; Benlamri, R. Extending Wireless Sensor Network Lifetime With Global Energy Balance. IEEE Sens. J. 2015, 15, 5053-5063. [CrossRef]

26. Li, G.Y.; Cao, Y.; Gao, X.; Tang, J. Energy balance routing protocol for wireless sensor networks based on fuzzy next-hop selection. Wuhan Univ. J. Nat. Sci. 2009, 14, 148-152. [CrossRef]

27. Ekal, H.H.; Abdullah, J.; Jamil, A.; Audah, L.; Alias, R. Energy balance mechanism for improving the lifetime in dense centric Wireless Sensor Networks. In Proceedings of the 7th Annual Information Technology, Electronics and Mobile Communication Conference (IEMCON), Vancouver, Canada, 13-15 October 2016; pp. 1-15. [CrossRef]

28. Xian, Q.; Zhang, W.T. Energy-Balanced Distance-Based Routing Algorithm in Wireless Sensor Networks. Appl. Mech. Mater. 2014, 539, 229-233. [CrossRef]

29. Mahfoudh, S.; Minet, P. Survey of Energy Efficient Strategies in Wireless Ad Hoc and Sensor Networks. In Proceedings of the 7th International Conference on Networking (ICN 2008), Cancun, Mexico, 13-18 April 2008; pp. 1-7. [CrossRef]

30. Long, Z.H.; Gao, M.J. Survey on network lifetime research for wireless sensor networks. In Proceedings of the 2nd IEEE International Conference on Broadband Network and Multimedia Technology, Beijing, China, 18-20 October 2009; pp. 899-902. [CrossRef]

31. Zawodniok, M.; Jagannathan, S. Predictive Congestion Control Protocol for Wireless Sensor Networks. IEEE Trans. Wirel. Commun. 2007, 6, 3955-3963. [CrossRef]

32. Ding, W.; Tang, L.R.; Ji, S.Y. Optimizing routing based on congestion control for wireless sensor networks. Wirel. Netw. 2016, 22, 915-925. [CrossRef]

33. Ding, W.; Tang, L.R.; Feng, S. Traffic-Aware and Energy-Efficient Routing Algorithm for Wireless Sensor Networks. Wireless Personal. Communication 2015, 85, 2669-2686. [CrossRef]

34. Rezaee, A.A. A Fuzzy Congestion Control Protocol Based on Active Queue Management in Wireless Sensor Networks with Medical Applications. Wireless Personal. Communication 2018, 98, 815-842. [CrossRef]

35. Tang, L.R.; Lu, Z.L.; Cai, J.Q.; Yan, J.Y. An Equilibrium Strategy-Based Routing Optimization Algorithm for Wireless Sensor Networks. Sensors 2018, 18, 3477. [CrossRef] [PubMed]

36. Tang, L.R.; Liu, H.T.; Yan, J.Y. Gravitation Theory Based Routing Algorithm for Active Wireless Sensor Networks. Wireless Personal. Communication 2017, 97, 269-280. [CrossRef]

37. Sun, Y.J.; Lin, C.L.; Jiang, H.F. An Energy Efficient Distributed Uneven Clustering Routing Algorithm for WSNs. Chin. J. Sens. Actuators 2015, 28, 1194-1200. [CrossRef] 
38. Jiang, H.F.; Qian, J.S.; Sun, Y.J.; Sun, R.K.; Li, J. Energy Cost Based Energy Optimized Routing Algorithm in WSN. Comput. Sci. 2012, 39, 73-76.

39. Zhang, D.G.; Li, G.; Zheng, K.; Ming, X.C.; Pan, Z.H. An Energy-Balanced Routing Method Based on Forward-Aware Factor for Wireless Sensor Networks. IEEE Trans. Ind. Inform. 2014, 10, 766-773. [CrossRef]

(c) (

(C) 2020 by the authors. Licensee MDPI, Basel, Switzerland. This article is an open access article distributed under the terms and conditions of the Creative Commons Attribution (CC BY) license (http://creativecommons.org/licenses/by/4.0/). 